\title{
DEMONSTRAÇÕES DO TEOREMA DE THALES: UM ENFOQUE HISTÓRICO
}

\author{
THALES' THEOREM DEMONSTRATIONS: A HISTORICAL FOCUS
}

\section{Ana Carolina Costa Pereira}

Universidade Estadual do Ceará

E-mail: carolina.pereira@uece.br

\begin{abstract}
Resumo
Muitos conceitos matemáticos que são estudados na Educação Básica estão permeados de aspectos históricos que muitos professores não têm conhecimento da sua existência. Esse artigo explora as ideias acerca da demonstração do teorema de Thales acerca de alguns momentos históricos: período pré-eudoxiano e pós-eudoxiano. Considera-se plausível que a ideia subjacente ao teorema de Thales ligado às condições de proporcionalidade de segmentos, isto é, medição de segmentos, pode ser uma forma de introduzir os números reais positivos. Assim, enfatizar o tratamento da comensurabilidade de segmentos ao desenvolver a demonstração do teorema de Thales, possibilita estabelecer uma relação com a construção dos Números Reais, acarretando uma possível proposta para seu tratamento, via medição.
\end{abstract}

Palavras-chave: teorema de Thales. história da matemática. números reais. educação matemática.

\begin{abstract}
Historic many teachers are unaware of its existence. This article explores the ideas about Thales theorem demonstration about some historical moments: pre-eudoxian and post-eudoxian. It is considered likely that the idea behind the Thales theorem connected to the proportionality conditions segments, ie segments measurement may be a way to introduce positive real numbers. Thus, emphasize the treatment of commensurability segments to develop the demonstration of Thales theorem, allows establish a relationship with the construction of the Real Numbers, leading to a possible proposal for its treatment via.
\end{abstract}

Keywords: Thales' theorem. history of mathematics. real numbers. mathematics education. 


\section{INTRODUÇÃO}

Um dos teoremas chave da Geometria Elementar é o conhecido teorema de Thales, que relaciona o geométrico e o numérico por meio de medidas. Seu enunciado mais conhecido atualmente é: Se um feixe de retas paralelas é cortado por duas transversais, então as medidas dos segmentos correspondentes que estão sobre a reta são diretamente proporcionais ${ }^{1}$.

Segundo a maioria dos autores de livros de história da Matemática, não se tem nenhuma evidencia histórica de quando e como surgiu o teorema de Thales, pois não existem documentos suficientes para comprovar sua existência e autoria. Muitas fontes sofreram alterações devido a várias versões de interpretações recebidas, dificultando separar o histórico do fantástico.

Contudo, conjectura-se que sua origem se deve à solução de problemas de natureza prática, principalmente na arquitetura e agrimensura grega, envolvendo paralelismo e proporcionalidade, relacionados diretamente ao geométrico e ao numérico. Provavelmente sua origem pode estar no método de medir a altura da pirâmide. Segundo Eves (2004),

há duas versões de como Tales calculou a altura de uma pirâmide egípcia por meio da sombra. O relato mais antigo, dado por Hierônimos, um discípulo de Aristóteles, diz que Tales anotou o comprimento da sombra no momento em que esta era igual à altura da pirâmide que a projetava. A versão posterior, dada por Plutarco, diz que ele fincou verticalmente uma vara e fez o uso da semelhança de triângulos. Ambas as versões pecam ao não mencionar a dificuldade de obter, nos dois casos, o comprimento da sombra da pirâmide - isto é, a distância da extremidade da sombra ao centro da base da pirâmide (EVES, 2004, p. 115).

As duas versões de Hierônimos e Plutarco para o modo de como Thales mediu a altura da pirâmide, expostas no livro de Eves (2004), podem fornecer dúvidas com relação ao feito. Na primeira versão, Thales teria medido a altura da pirâmide pela observação de sua sombra com a sombra da pirâmide, porém, medir a altura da pirâmide tomando apenas essas variáveis, poderia ocasionar erro na medida, pois ele teria de levar em consideração sua posição, o horário do dia, a época do ano, a latitude etc., o que, em nenhum momento, foi mencionado na descrição. Isso 
acontece também com a versão de Plutarco. Portanto, é importante, ao referirmos esses tipos de fatos históricos, levarmos em consideração todas as possíveis condições de realização dos feitos.

O teorema de Thales, que enuncia as condições de proporcionalidade de segmentos, até o final do século XIX era conhecido apenas como teorema das Linhas Proporcionais. Segundo Massot (1995), na França, nesse mesmo período, alguns autores como Rouche e Comberousse, no livro Éléments de Géométrie, foram pioneiros nessa designação.

No Brasil, o aparecimento do nome teorema de Thales relacionado ao teorema da Proporcionalidade surgiu na segunda metade do século $X X$, principalmente nos livros-texto que caracterizaram o Movimento da Matemática Moderna, como o livro do autor Osvaldo Sangiorgi. A partir desse momento começou a surgir uma variedade de enunciados referentes ao teorema da Thales.

Outros autores, anteriores aos dessa época, fazem referência ao teorema de Thales, ligando-o a um resultado do conteúdo de semelhança de triângulos. O livro da FIC, Elementos de Geometria, datado do início do século XX, enuncia da seguinte forma: "Toda parallela a um lado d’um triângulo determinam segundo triângulo semelhante ao primeiro" (FIC, 1923, p. 93). O livro de autoria de Ary Quintella, Matemática: Curso Ginasial enuncia como lei de Thales "a paralela traçada a um dos lados de um segundo triângulo semelhante ao primeiro" (QUINTELLA,1963).

Uma pesquisa, realizada por Massot (1995) para a Institut de Recherche sur l'Enseignement des Mathématiques - IREM, mostra as várias enunciações do teorema em diversos países:

Na Itália: "I segmenti staccati da um fascio di rette parallele su due trasversali sono direttamente proporzionali" (Os segmentos determinados por um feixe de paralelas sobre duas transversais são diretamente proporcionais).

$\mathrm{Na}$ Alemanha o teorema de Thales é enunciado como "todo triângulo inscrito numa semicircunferência é retângulo"; porém o teorema que corresponde ao teorema de Thales

\footnotetext{
${ }^{1}$ Adotaremos essa versão quando nos referirmos ao teorema de Thales.
} 
utilizado no Brasil é conhecido como Teorema dos Segmentos Proporcionais: "Se um feixe de retas concorrentes é cortado por duas retas paralelas, então a razão entre as medidas dos segmentos determinados por uma reta do feixe é igual à razão entre as medidas dos segmentos correspondentes determinados sobre qualquer outra reta do feixe".

$\mathrm{Na}$ Espanha, "Si cortamos dos rectas cualesquiera, por varias rectas paralelas, los segmentos correspondientes determinados em ambias, son proporcionales". (Se cortamos duas retas quaisquer por várias retas paralelas, os segmentos correspondentes determinados em ambas, são proporcionais).

Deste modo, esse artigo tem o intuito de explorar as ideias acerca da demonstração do teorema de Thales, em textos diversos, que convergem ou divergem para ideias acerca de alguns momentos históricos: período pré-eudoxiano, na descoberta da teoria das proporções de Eudoxo que inclui da demonstração euclidiana.

Tendo em vista que os pitagóricos e Eudoxo tiveram seus estudos transmitidos por meio da tradição oral e só foram registrados, por outros autores, séculos mais tarde, voltamo-nos para o mais expressivo desses autores a fim de discutir o conteúdo, a forma e a coerência desses registros com as informações que se tinha daquela época.

A obra Os Elementos de Euclides, mais precisamente os livros V e VI, será estudada e discutida, sendo apontados fatores relevantes à compreensão, tanto dos momentos que ele pretendia descrever, quanto das conveniências encontradas na descrição.

Por fim, olhando para a produção do conhecimento como um movimento histórico, deparamo-nos com um esforço humano, através do tempo, de sistematização dos estudos desenvolvidos para que outros, num outro tempo, possam compreendê-los ou complementá-los. Essas ideias estão, a nosso ver, imersas em outras, que têm como fundamento o conhecimento como algo universal. 


\section{DEMONSTRAÇÕES DO TEOREMA DE THALES}

A ideia por trás do teorema de Thales está ligada às condições de proporcionalidade de segmentos, em que esses segmentos podem ser comensuráveis ou incomensuráveis com uma unidade de medição. No Ensino Fundamental e Médio, muitos livros didáticos atuais demonstramno, trabalhando, apenas, com o caso em que os segmentos (grandezas) são comensuráveis ${ }^{2}$ como provavelmente os pitagóricos trabalhavam, associando a um número inteiro ou uma razão entre dois números inteiros. Uma exposição organizada da Matemática pitagórica está nos livros VII, VIII e IX dos Elementos de Euclides.

Contudo, o conceito de proporção, para os gregos, estava ligado à ideia de subtração mútua. Segundo Boyer (1998),

aparentemente os gregos usaram a idéia de que quatro quantidades estão em proporção $a: b=c: d$, se as duas razões a:b e c:d têm a mesma subtração mútua; isto é, se em cada razão a quantidade menor cabe um igual número inteiro de vezes na menor e o novo resto no precedente o mesmo número inteiro de vezes, e assim por diante. (BOYER, 1998, p. 61).

Essa citação nos mostra que, sendo a razão a:b, na qual a grandeza a é um múltiplo da grandeza b, por exemplo, $a=8$ e $b=2$, isso significará que $\mathbf{b}$ caberá exatamente quatro vezes em $\mathbf{a}$; então, a outra razão c:d só será igual a a:b, se apresentar a mesma propriedade, ou seja d coube exatamente quatro vezes em $\mathbf{c}$. Assim, após subtrairmos quatro vezes $\mathbf{b}$ de $\mathbf{a}$ ou $\mathbf{d}$ de $\mathbf{c}$, os restos, em cada caso, seriam iguais a zero.

Outro caso seria se a divisão não fosse exata. Quando isso ocorrer poder-se-á utilizar o processo das divisões sucessivas para determinação do Máximo Divisor Comum (MDC) entre dois números.

À medida que a Matemática se desenvolvia, as grandezas incomensuráveis se tornaram um problema, gerando o que se costuma chamar de "a crise dos incomensuráveis".

\footnotetext{
${ }^{2}$ Dois segmentos $\overline{\mathrm{AB}}$ e $\overline{\mathrm{CD}}$ são comensuráveis se existem um segmento $\mathrm{U}$ e dois inteiros $m$ e $\mathrm{n}$ tais que $\mathrm{AB}=\mathrm{m}$.U e $\mathrm{CD}$ $=n \cdot U$
} 
Uma referência sobre a descoberta dos incomensuráveis pode ser encontrada no trabalho de Aristóteles, citado por Fowler (1999), que traz uma prova da incomensurabilidade da diagonal de um quadrado, com o seu lado indicando que se baseava na distinção entre pares e ímpares. Ele ainda menciona a ilustração Matemática favorita de Aristóteles para a incomensurabilidade, a da diagonal do quadrado, embora ele nunca sugira o fato que o levou à descoberta. Porém, o próprio Fowler (1999) discute que esse problema relacionado à diagonal do quadrado é muito simples para os pitagóricos, e que o problema surgiu da observação das diagonais de um pentágono, pois quando se traçam as cinco diagonais de um pentágono, elas formam um pentágono regular menor. As diagonais desse segundo pentágono, por sua vez, formam um terceiro pentágono regular, que é ainda menor. Esse processo pode continuar indefinidamente, resultando em pentágonos tão pequenos quanto se queira, levando à conclusão que a razão da diagonal para o lado, num pentágono regular, não é racional. E, ainda, acredita que os pitagóricos já conheciam o problema da incomensurabilidade logo no início de suas investigações Matemáticas, mas a mantinham como um segredo da seita.

O principal perigo da teoria dos incomensuráveis é que ela destruiria a generalidade da teoria das proporções, que só continuaria válida para grandezas comensuráveis. Segundo Fowler (1999), não há referências claras das tentativas feitas pelos pitagóricos para resolver esse impasse. Ele ainda ressalta que "até de onde eu sei, nenhum texto grego, cedo ou tarde, nos conta claramente sobre as dificuldades Matemáticas elevadas pela incomensurabilidade" (FOWLER, 1999, p. 363).

A descoberta forçou os pitagóricos a abandonar a sua filosofia básica de que todas as coisas eram números, e permitiu que os matemáticos gregos desenvolvessem novas teorias. O problema não foi resolvido antes da descoberta da teoria das proporções de Eudoxo de Cnido (408 a.C. - 355 a.C.) encontrado no livro $\mathrm{V}$ de $O$ s Elementos de Euclides, em que a autenticidade do fato seria concluída, segundo Lintz, por citações de Arquimedes.

Lintz (1999) retrata que

o trágico resultado da noção de grandezas incomensuráveis foi o descrédito da Matemática e dos matemáticos que, no final das contas, construíram um edifício 
sobre a areia e não faltaram críticos violentos que expuseram os pitagóricos ao ridículo. Mas, apesar de tudo, a pesquisa continuou e resultados continuaram a se acumular até o advento de Eudóxio que, finalmente, introduziu uma teoria correta dos incomensuráveis, reabilitando a Matemática como "ciência exata", inaugurando o período da arte (LINTZ,1999, p. 83).

O livro $\mathrm{V}$ de Os Elementos começa assumindo, na primeira definição, o conceito de magnitude como um conceito primitivo, que teria profundas raízes na imagem grega do universo. Alertamos para o equívoco de procurar entender essas discussões como um grego e não como um matemático atual. Como primeira aproximação, do ponto de vista matemático, podemos considerar magnitude como algo que pode ser aumentado, diminuído ou agregado a outros objetos da mesma espécie, como por exemplo, um segmento, uma superfície, um número.

O essencial da noção de magnitude ou grandeza seria a possibilidade de encontrar seus múltiplos. Dos geômetras gregos não chegou até nós nenhuma informação sobre a maneira de introduzir, com precisão, esses conceitos.

Eudoxo define, também, a igualdade de razões, que se aplica a grandezas comensuráveis ou incomensuráveis e contém a ideia de proporcionalidade:

“Diz-se que quatro grandezas estão na mesma razão, a primeira para a segunda e a terceira para a quarta se, quando eqüimúltiplos quaisquer são tomados da primeira e da terceira e eqüimúltiplos quaisquer da segunda e da quarta, os primeiros eqüimúltiplos são ambos maiores que, ou ambos iguais a, ou ambos menores que, os últimos eqüimúltiplos considerados em ordem correspondente (Definição 5 do V Livro de Os Elementos de Euclides) ${ }^{3 \prime \prime}$.

\footnotetext{
3 "Magnitudes are said to be in the same ratio, the first to second and the third to the fourth, when, if any equimultiples whatever be taken of the first and third, and any equimultiples whatever of the second and fourth, the former equimultiples alike exceed, are alike equal to, or alike fall short of, the latter equimultiples respectively taken in corresponding order" (HEATH, 1956, p 114).
} 
Em linguagem atual, considerando como grandezas os segmentos $A D, D B, A E$ e EC (comensuráveis ou não), dizer que $\frac{A D}{D B}=\frac{A E}{E C}$, significa dizer que, para todo $\mathrm{m}$ e $\mathrm{n}$ inteiros positivos, as seguintes condições se verificam:

Se $m . D B<n$. $A D$, então $m . E C<n . A E$

Se $m . D B>n$. $A D$, então $m$. $E C>n$. $A E$

Se $m . D B=n . A D$, então $m . E C=n . A E$.

No livro VI, é feito o estudo da semelhança de figuras, a começar pela proposição fundamental, relacionada à proporcionalidade de segmentos determinados em duas retas cortadas por um feixe de paralelas, cuja demonstração dada pelos pitagóricos era incompleta, porque dependia da comensurabilidade das grandezas envolvidas ${ }^{4}$.

Uma das consequências, supostamente causadas pela teoria das proporções de Eudoxo, foi forçar a forte separação entre números e geometria, deixando somente à geometria o tratamento das razões incomensuráveis. Os gregos foram um tanto afastados de um desenvolvimento numérico da Matemática. A aritmética e a álgebra só voltariam a ganhar importância e autonomia próprias com a influência árabe, a partir do século XII.

Outro livro de Os Elementos, em que podemos encontrar discussões sobre comensurabilidade de grandezas, é o Livro X. Nele está exposta a teoria geral das grandezas incomensuráveis, que, segundo Lintz (1999, p. 161), é "considerado pela tradição como o mais perfeito e bem acabado de todos eles". O livro inicia-se com quatro definições, desencadeando 115 proposições relacionadas à teoria dos incomensuráveis.

A questão referente à existência de certa grandeza e à inexistência de um número (racional) que o representasse só é retomada e encaminhada por Dedekind, no século XIX. Ele percebe, na teoria das Proporções de Eudoxo, a possibilidade de pensar na essência da continuidade de um segmento a partir de sua divisão em duas classes. A insuficiência dos números naturais é percebida pela correspondência não biunívoca entre estes e os pontos de uma reta tomada contínua. A superação

\footnotetext{
${ }^{4}$ Essa proposição fundamental será demonstrada nas próximas seções, pois é considerado por nós o teorema da Proporcionalidade de Segmentos ou Teorema de Thales exposto no Livro V dos Elementos de Euclides.
} 
dessa insuficiência é alcançada pela inclusão de outro conjunto numérico que, unido ao anterior, daria origem ao conjunto dos números reais.

\section{PERÍODO PRÉ-EUDOXIANO}

Apesar das dúvidas sobre a veracidade dos feitos de Thales (calcular a altura da pirâmide, a largura de um rio, a distância de um barco se aproxima, etc.), citado anteriormente, segundo Lintz (1999, p. 77) "fica certo que em sua época já eram bem difundidas as principais propriedades dos triângulos e a teoria da proporção que se baseia no resultado bem conhecido: se duas retas $a$ e $b$ são cortadas por retas paralelas, os vários segmentos determinados em a e b são proporcionais, isto é $\frac{A B}{A^{\prime} B^{\prime}}=\frac{B C}{B^{\prime} C^{\prime}}=\ldots$ ".. (LINTZ, 1999, p. 77).

Assim, com essa afirmação podemos acreditar que a proposição, hoje intitulada teorema de Thales, já era conhecida de longa data, embora sem prova severa. A demonstração rigorosa desse teorema só vai aparecer com Euclides, baseado na teoria dos incomensuráveis de Eudoxo. Uma ideia defendida por Lintz (1999) é a de que o problema proposto na proposição anterior foi à primeira tentativa de sistematizar a geometria.

No livro História da Matemática, Lintz (1999) apresenta duas demonstrações para o teorema. Na primeira, o autor se baseia no conceito de números na época de Thales, em que "número é uma coleção de unidades e, por sua vez, unidade é um ponto sem posição" (LINTZ, 1999, p. 59). Vejamos a demonstração apresentada por ele:

Proposição: Se duas retas $\underline{a}$ e $\underline{b}$ são cortadas por retas paralelas, os vários segmentos determinados em a e $\underline{b}$ são proporcionais, isto é $\frac{A B}{A^{\prime} B^{\prime}}=\frac{B C}{B^{\prime} C^{\prime}}=\ldots$ (LINTZ, 1999: 39). 
Figura 1. Demonstração.
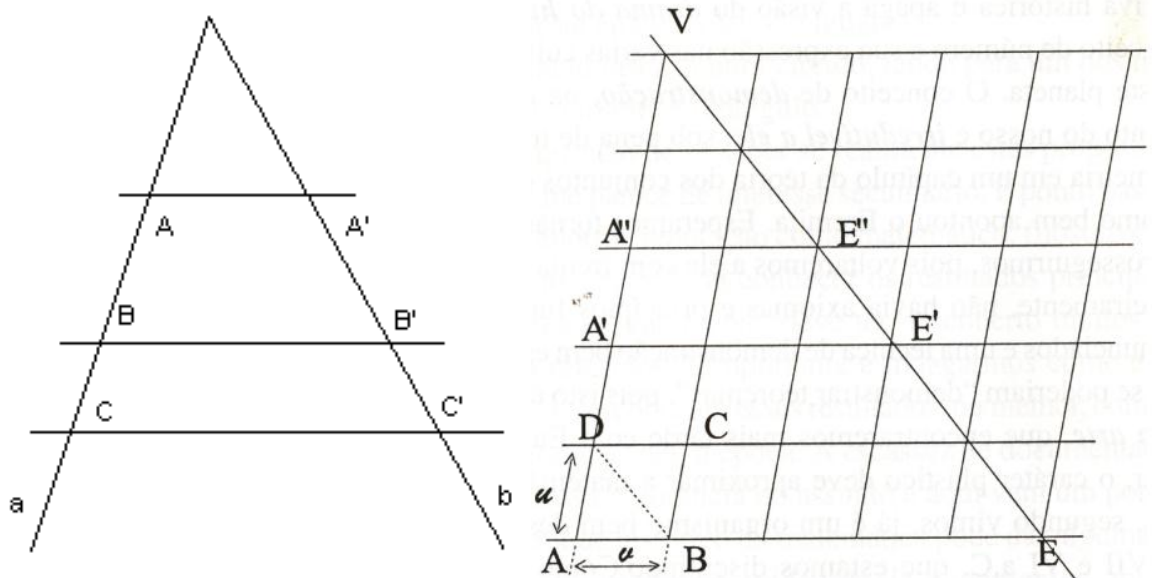

Fonte: Lintz, 1999.

\section{Demonstração}

Tomemos um segmento u como unidade e construamos o reticulado da figura 2, formado de dois feixes de retas paralelas, definindo o quadrilátero $A B C D$, etc., (figura 2) de lados u e v. Consideremos as duas retas VA e VE e as paralelas AE, $A^{\prime} E^{\prime}, A^{\prime \prime} E^{\prime \prime}$. Então, diretamente da figura, vem
$V A^{\prime \prime}=2 u$
$V E^{\prime \prime}=2 W$
$V A^{\prime}=3 u$
$V^{\prime}=3 w$

onde w é o segmento DB tomado como unidade de medida das retas paralelas a DB.

(...)

Das relações acima, tiramos

$\frac{V A^{\prime \prime}}{V A^{\prime}}=\frac{2}{3}=\frac{V E^{\prime \prime}}{V E^{\prime}}$

O resultado facilmente se generaliza para a situação da figura 2 , desde que os segmentos determinados nas retas a e $\underline{b}$ sejam múltiplos, respectivamente, de unidades $\underline{u}$ e $\underline{v}$ pré-estabelecidas. Esta hipótese da existência de unidade comum para medir segmentos está ligada à questão da comensurabilidade de segmentos de grande profundidade e importância para a geometria e que vai culminar com a crise da escola pitagórica (...) (LINTZ, 1999, p. 42-43). 
Outra tentativa de demonstração era a utilização das noções de números, na época dos pitagóricos, para o problema da proporcionalidade, o qual era exposto da seguinte maneira:

Proposição: Se duas retas a e b são cortadas por um certo número de paralelas, estas determinavam sobre a e b segmentos proporcionais, isto é, $\frac{V A}{V A^{\prime}}=\frac{A B}{A^{\prime} B^{\prime}}=\frac{B C}{B^{\prime} C^{\prime}} \cdot($ LINTZ, 1999: 63)

Figura 1. Demonstração.
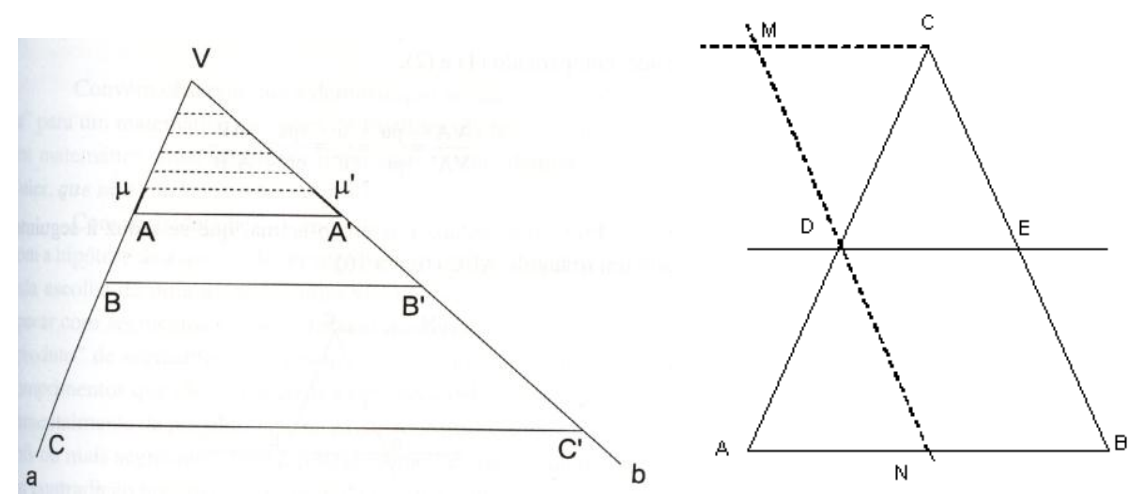

Fonte: Lintz, 1999.

De acordo com a hipótese fundamental acima enunciada ${ }^{5}$ existe uma unidade ou módulo u em a tal que

$$
V A=p \times u ; A B=q \times u(1)
$$

Assim, VA fica subdividida em $\mathbf{p}$ segmentos e $A B$ em $\mathbf{q}$ segmentos. $O$ teorema ficará demonstrado se provarmos que, traçando-se as paralelas tracejadas a $A A^{\prime}$ e $B B^{\prime}$, também $V A^{\prime}$ e $A^{\prime} B^{\prime}$ ficam subdivididos no mesmo número de partes iguais e, neste caso, se $\mathbf{u}^{\prime}$ é uma dessas partes teremos $V A^{\prime}=p \cdot u^{\prime} ; A^{\prime} B^{\prime}=q \cdot u^{\prime}(2)$ donde, comparando (1) e (2),

$$
\frac{V A}{V A^{\prime}}=\frac{p u}{p u^{\prime}}=\frac{u}{u^{\prime}}=\frac{q u}{q u^{\prime}}=\frac{A B}{A^{\prime} B^{\prime}}
$$

\footnotetext{
${ }^{5}$ A hipótese fundamental que Lintz refere é: seja u um segmento unidade ou módulo e seja $A B$ um segmento qualquer. 1) u pode ser aplicado um número inteiro $\boldsymbol{n}$ vezes sobre $A B$, então diremos que $A B$ é $\boldsymbol{n}$ vezes $\mathbf{u}$ ou $A B=n \cdot u$ (...) e 2) u não pode ser aplicado um número inteiro de vezes sobre $A B$ e, então subdividimos $\mathbf{u}$ em $\mathbf{q}$ partes novamente. Pode acontecer que $A B$ seja igual a $\mathbf{p}$ vezes o novo módulo ou nova unidade $u / q$ ou não, caso em que
} 
Provemos, então, a asserção acima, que se reduz à seguinte: dado um triângulo $A B C$ (figura 4) e o ponto médio $D$ de um dos lados, se traçarmos por $D$ uma paralela a $A B$, ela divide $C B$ em duas partes iguais $C E$ e $E B$. De fato, tracemos por $D$ uma paralela a $\mathrm{CB}$ e por $\mathrm{C}$, uma paralela a $\mathrm{AB}$. Agora usaremos o fato de serem iguais segmentos determinados em duas paralelas cortadas por outras duas. Os pitagóricos seguramente conheciam este fato que, nos elementos de Euclides, pode ser obtido facilmente do teorema sobre ângulos alternos e igualdade de triângulos, mas é provável que eles o conhecessem através de "demonstrações incompletas", quando comparados ao padrão de Euclides. Enfim, assumindo isso, os triângulos DMC e AND são iguais e, portanto, $\mathrm{DM}=\mathrm{DN}$; mas $\mathrm{DM}=\mathrm{CE}$ e $\mathrm{DN}=$ $E B$, logo CE = EB. q.e.d (LINTZ, 1999, p. 63-65).

Nota-se uma mudança razoável da demonstração da época de Thales para a época de Pitágoras, embora ambas estejam incompletas. Enquanto Thales tira conclusões a partir das figuras, tomando-as como hipótese válida para o raciocínio da demonstração, os pitagóricos vão além, conseguem assimilar certa organização para a época, desprendendo-se de algumas particularidades visuais das figuras.

\section{APÓS A DESCOBERTA DA TEORIA DAS PROPORÇÕES DE EUDOXO}

\subsection{Demonstração pela Teoria da Proporção}

Até antes da descoberta da Teoria das Proporções, trabalhava-se apenas com o caso em que os segmentos (grandezas) eram comensuráveis, associado a número natural ou a uma razão de dois números naturais. Entretanto, após a descoberta do problema das grandezas incomensuráveis, muitos dos matemáticos gregos voltaram-se para solucionar o problema das grandezas incomensuráveis, desenvolvendo, assim, novas teorias. Esse problema foi resolvido pela descoberta da teoria das proporções de Eudoxo de Cnido (408 a.C. - 355 a.C.), encontrado no Livro V de Os Elementos de Euclides. Uma das consequências, supostamente causadas pela Teoria das Proporções de Eudoxo, foi forçar uma forte separação entre números e geometria, deixando somente à geometria o tratamento das razões incomensuráveis.

repetiremos o processo usado agora u/q dividido em q' partes como módulo e assim sucessivamente (Lintz, 1999, p. 60-61). 
Para provar o teorema de Thales utilizando a teoria das proporções devemos considerar os segmentos $\overline{\mathrm{AB}}, \overline{\mathrm{BC}}, \overline{\mathrm{A}^{\prime} \mathrm{B}^{\prime}}, \overline{\mathrm{B}^{\prime} \mathrm{C}^{\prime}}$ (comensuráveis ou não), em que $\frac{\overline{A B}}{\overline{B C}}=\frac{\overline{A^{\prime} B^{\prime}}}{\overline{B^{\prime} C^{\prime}}}$ é válida, se as três condições abaixo, para todo m e n naturais quaisquer, forem satisfeitas:

Se $\mathrm{n} \overline{A B}<\mathrm{m} \overline{B C} \Rightarrow \mathrm{n} \overline{A^{\prime} B^{\prime}}<\mathrm{m} \overline{B^{\prime} C^{\prime}}$;

Se $\mathrm{n} \overline{A B}=\mathrm{m} \overline{B C} \Rightarrow \mathrm{n} \overline{A^{\prime} B^{\prime}}=\mathrm{m} \overline{B^{\prime} C^{\prime}}$;

Se $\mathrm{n} \overline{A B}>\mathrm{m} \overline{B C} \Rightarrow \mathrm{n} \overline{A^{\prime} B^{\prime}}>\mathrm{m} \overline{B^{\prime} C^{\prime}}$.

Figura 3. Demonstração.

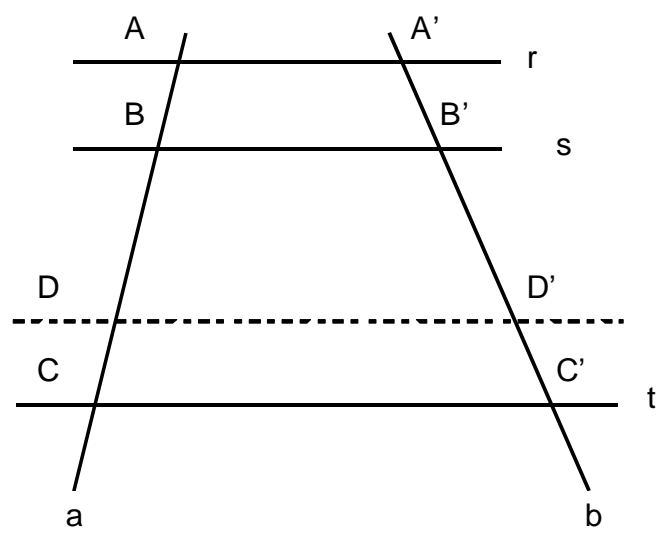

Fonte: dados da pesquisa.

Teorema: Se um feixe de retas paralelas é cortado por duas transversais, então as medidas dos segmentos correspondentes que estão sobre a reta são diretamente proporcionais.

\section{Demonstração}

Assim, tomaremos $m$ e $n$ dois números naturais quaisquer, iremos dividir o segmento $\overline{\mathrm{AB}}$ em $\mathrm{m}$ partes iguais de um certo segmento $U$, então teremos $\overline{\mathrm{AB}}=\mathrm{mU}$ e traçando-se paralelas dividiremos $\overline{A^{\prime} B^{\prime}}$ em $m$ partes iguais de um certo $U^{\prime}$, de modo que $\overline{A^{\prime} B^{\prime}}=m U^{\prime}$. Na reta a, partindo de $B$ para $C$, marcaremos $n$ segmentos $\mathrm{U}(\overline{B C}=n U)$. Do mesmo modo na reta $\mathbf{b}$, partindo de $\mathrm{B}^{\prime}$ para $C^{\prime}$, marcaremos $n$ segmentos $U^{\prime}\left(\overline{B^{\prime} C^{\prime}}=n U^{\prime}\right)$. Sendo $\mathrm{D}$ a ultima extremidade do último segmento contido em $\overline{B C}$ podemos ter três casos possíveis: 
10 caso: D está entre $\mathrm{B}$ e $\mathrm{C}$ (provar $\mathrm{n} \overline{A B}<\mathrm{m} \overline{B C} \Rightarrow \mathrm{n} \overline{A^{\prime} B^{\prime}}<\mathrm{m} \overline{B^{\prime} C^{\prime}}$.);

20 caso: $\mathrm{D}$ coincide com $\mathrm{C}\left(\mathrm{n} \overline{A B}=\mathrm{m} \overline{B C} \Rightarrow \mathrm{n} \overline{A^{\prime} B^{\prime}}=\mathrm{m} \overline{B^{\prime} C^{\prime}}\right.$.);

3o caso: D está além de $\mathrm{C}\left(\mathrm{n} \overline{A B}>\mathrm{m} \overline{B C} \Rightarrow \mathrm{n} \overline{A^{\prime} B^{\prime}}>\mathrm{m} \overline{B^{\prime} C^{\prime}}\right.$.).

Analisando o 10 caso em que D está entre B e C.

De $\overline{A B}=\mathrm{mU}$ temos que $\mathrm{n} \overline{A B}=\mathrm{nmU}$ e de $\overline{B D}=\mathrm{nU}$ temos que $\mathrm{m} \overline{B D}=\mathrm{mnU}$. Logo $\mathrm{n} \overline{A B}=\mathrm{m} \overline{B D}$. Como $\overline{B D}<\overline{B C}$ então $\mathrm{n} \overline{A B}=\mathrm{m} \overline{B D}<\mathrm{m} \overline{B C}$. Portanto $\mathrm{n} \overline{A B}<\mathrm{m} \overline{B C}$.

Por outro lado, de $\overline{A^{\prime} B^{\prime}}=\mathrm{m} U^{\prime}$ temos que $\mathrm{n} \overline{A^{\prime} B^{\prime}}=\mathrm{nm} \mathrm{U}^{\prime}$ e de $\overline{B^{\prime} D^{\prime}}=\mathrm{nU} \mathrm{U}^{\prime}$ temos que $\mathrm{m} \overline{B^{\prime} D^{\prime}}=$ $\mathrm{mnU}$ ". Logo $\mathrm{n} \overline{A^{\prime} B^{\prime}}=\mathrm{m} \overline{B^{\prime} D^{\prime}}$.

Como $\overline{B^{\prime} D^{\prime}}<\overline{B^{\prime} C^{\prime}}$ entãon $\overline{A^{\prime} B^{\prime}}=\mathrm{m} \overline{B^{\prime} D^{\prime}}<\mathrm{m} \overline{B^{\prime} C^{\prime}}$. Portanto $\mathrm{n} \overline{A^{\prime} B^{\prime}}<\mathrm{m} \overline{B^{\prime} C^{\prime}}$.

Concluímos que $\mathrm{n} \overline{A B}<\mathrm{m} \overline{B C} \Rightarrow \mathrm{n} \overline{A^{\prime} B^{\prime}}<\mathrm{m} \overline{B^{\prime} C^{\prime}}$.

O 2 caso em que D coincide com $C$.

De $\overline{A B}=\mathrm{mU}$ temos que $\mathrm{n} \overline{A B}=\mathrm{nmU}$ e de $\overline{B D}=\mathrm{nU}$ temos que $\mathrm{m} \overline{B D}=\mathrm{mnU}$. Logo $\mathrm{n} \overline{A B}=\mathrm{m} \overline{B D}$.

De $\overline{A^{\prime} B^{\prime}}=\mathrm{mU} U^{\prime}$ vem que $\mathrm{n} \overline{A^{\prime} B^{\prime}}=\mathrm{nmU}$ e de $\overline{B^{\prime} C^{\prime}}=\mathrm{n} U^{\prime}$ vem que $\mathrm{m} \overline{B^{\prime} C^{\prime}}=\mathrm{mnU}$. Logo $\mathrm{n} \overline{A^{\prime} B^{\prime}}=$ $\mathrm{m} \overline{B^{\prime} C^{\prime}}$

Concluímos que $\mathrm{n} \overline{A B}=\mathrm{m} \overline{B C} \Rightarrow \mathrm{n} \overline{A^{\prime} B^{\prime}}=\mathrm{m} \overline{B^{\prime} C^{\prime}}$.

O 3ํ caso em que D está além de C.

De $\overline{A B}=\mathrm{mU}$ temos que $\mathrm{n} \overline{A B}=\mathrm{nmU}$ e de $\overline{B D}=\mathrm{nU}$ temos que $\mathrm{m} \overline{B D}=\mathrm{mnU}$. Logo $\mathrm{n} \overline{A B}=\mathrm{m} \overline{B D}$

Como $\overline{B D}>\overline{B C}$ então $\mathrm{n} \overline{A B}=\mathrm{m} \overline{B D}>\mathrm{m} \overline{B C}$. Logo $\mathrm{n} \overline{A B}>\mathrm{m} \overline{B C}$.

De $\overline{A^{\prime} B^{\prime}}=\mathrm{m} U^{\prime}$ temos que $\mathrm{n} \overline{A^{\prime} B^{\prime}}=\mathrm{nmU} U^{\prime}$ e de $\overline{B^{\prime} D^{\prime}}=\mathrm{n} U^{\prime}$ então $\mathrm{m} \overline{B^{\prime} D^{\prime}}=\mathrm{mnU}$. Logo $\mathrm{n} \overline{A^{\prime} B^{\prime}}=$ $\mathrm{m} \overline{B^{\prime} D^{\prime}}$.

Como $\overline{B^{\prime} D^{\prime}}>\overline{B^{\prime} C^{\prime}}$ entãon $\overline{A^{\prime} B^{\prime}}=\mathrm{m} \overline{B^{\prime} D^{\prime}}>\mathrm{m} \overline{B^{\prime} C^{\prime}}$. Logo $\mathrm{n} \overline{A^{\prime} B^{\prime}}>\mathrm{m} \overline{B^{\prime} C^{\prime}}$.

Concluímos que $\mathrm{n} \overline{A B}>\mathrm{m} \overline{B C} \Rightarrow \mathrm{n} \overline{A^{\prime} B^{\prime}}>\mathrm{m} \overline{B^{\prime} C^{\prime}}$.

Portanto, as três condições estão satisfeitas, provando que $\frac{\overline{A B}}{\overline{B C}}=\frac{\overline{A^{\prime} B^{\prime}}}{\overline{B^{\prime} C^{\prime}}}$. 


\subsection{Demonstração Euclidiana}

A demonstração euclidiana ${ }^{6}$, retirada do livro VI de Os Elementos de Euclides, baseia-se no método das áreas e na teoria das proporções.

O método das áreas consiste na junção do postulado das paralelas e na equivalência de triângulos, expostas no livro I dos "Os Elementos". As proposições 37, 38 e 39 estabelecem ideias relacionadas a esses conceitos, a partir dos quais são enunciadas e demonstradas. Segundo Haruna (2000),

O método das áreas permite afirmar a igualdade de duas superfícies. O problema surge ao comparar duas áreas e exprimir essa relação, isto é, combinar ao mesmo tempo cada uma dessas áreas com uma cota comum, surgindo o problema das grandezas comensuráveis e incomensuráveis (HARUNA, 2000, p. 14).

Quando as grandezas são comensuráveis, a proposição 1 do Livro $\mathrm{VI}$, dos "Os Elementos" de Euclides, fica bem definida, mas, quando as grandezas são incomensuráveis, surge a necessidade de usar a teoria da proporção explicada anteriormente.

Quando utilizamos a teoria das proporções associada ao método das áreas pela proposição 38 do Livro I, temos:

$$
\begin{aligned}
& \text { área } A C E=m \text { áreas } A C B \\
& \text { área } A C F=m \text { áreas } A C D
\end{aligned}
$$

Figura 4. Demonstração.

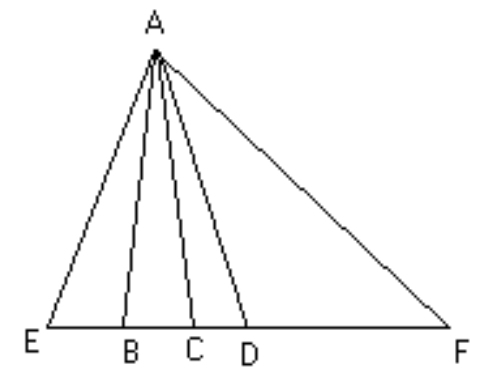

Fonte: dados da pesquisa. 
Assim, pode-se mostrar facilmente que se $\overline{\mathrm{CE}}$ é maior, igual, ou menor que $\overline{\mathrm{CF}}$, então a área do triângulo ACE é maior que, igual a ou menor que a área do triângulo de ACF. Em outras palavras:

$m \mathrm{BC}>\mathrm{n} C \mathrm{C}$ implica $\mathrm{m}$ área $\mathrm{ACB}>\mathrm{n}$ áreas $\mathrm{ACD}$

$\mathrm{m} B C=\mathrm{n} C D$ implica $\mathrm{m}$ área $\mathrm{ACB}=\mathrm{n}$ áreas $\mathrm{ACD}$

$\mathrm{m} B C<\mathrm{n} C D$ implica $\mathrm{m}$ área $\mathrm{ACB}<\mathrm{n}$ áreas $\mathrm{ACD}$

Então, a razão de $\overline{\mathrm{BC}}$ para $\overline{\mathrm{CD}}$ é a mesma que a da área do triângulo de $\mathrm{ABC}$ no triângulo de $\mathrm{ACD}$.

Apresentados alguns conceitos necessários para a demonstração, vejamos agora a enunciação e a demonstração da proposição 2, do Livro VI dos "Os Elementos" de Euclides, conhecido como teorema de Thales:

Proposição 2: Se uma linha direta é desenhada paralela a um dos lados de um triângulo, cortará os lados do triângulo proporcionalmente; e se os lados do triângulo sejam cortados proporcionalmente, a linha que une os pontos da secção será paralelo ao lado restante do triângulo.

Figura 5. Demonstração.

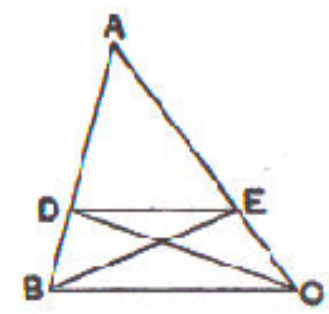

Fonte: dados da pesquisa.

\section{Demonstração':}

Seja $D E$ paralelo $A B C$, um dos lados do triangulo $A B C$.

Eu digo que, como BD está para DA, então CE está para EA. Seja BE e CD traçados. Então a triângulo $\mathrm{BDE}$ é igual ao triângulo $\mathrm{CDE}$, porque eles estão na mesma base $\mathrm{DE}$ e nas

\footnotetext{
${ }^{6}$ Primeira demonstração conhecida do Teorema de Thales surgiu três séculos depois da existência de Thales, localizada na proposição 2 do Livro VI dos “Os Elementos" de Euclides (300 a.C.), sustentada na teoria da proporção de Eudoxo, apresentada no Livro $V$ de Euclides.

7 Tentamos fazer uma tradução, exposta no Livro VI, dos “Os Elementos” de Euclides (HEATH, 1956), da demonstração da proposição 2, designada por nós teorema de Thales.
} 
mesmas paralelas DE e BC (proposição 38 do livro I) ${ }^{8}$. O triângulo ADE Possui uma outra área, mas possuem a mesma razão (Proposição 7 do livro $\mathrm{V}$ ) ${ }^{9}$ então como o triângulo BDE está para o triângulo $A D E$, então o triângulo $C D E$ está para o triângulo $A D E$.

Mas, como o triângulo $\mathrm{BDE}$ está para $\mathrm{ADE}$, então $\mathrm{BD}$ está para $\mathrm{DA}$; pois estando sobre a mesma altura, a perpendicular traçada de $E$ para $A B$, estão para um outro, como suas bases (proposição 1 do livro VI).

Pela mesma razão, como o triângulo CDE está para $A D E$, então CE está para $E A$.

Portanto temos também, como BD está para DA, então CE está para EA (proposição 2 do livro $V)^{10}$.

Por outro lado, vamos cortar os lados $A B$ e $C A$ do triângulo $A B C$ proporcionalmente, de forma que BD está para DA, como CE está para EA; e seja DE traçado.

Eu digo que DE é paralelo a BC.

Para, com a mesma construção, desde que BD está para $\mathrm{DA}$, como CE está para $\mathrm{EA}$, mas como $B D$ está para $D A$, então está o triângulo $B D E$ para o triângulo $A D E$, e como $C E$ está para EA, então está o triângulo CDE para o triângulo ADE (proposição 1 do Livro VI).

Além disso, como o triângulo $\mathrm{BDE}$ esta para o triângulo $\mathrm{ADE}$, então está o triângulo $\mathrm{CDE}$ para o triângulo ADE (proposição 2 do Livro V).

Portanto cada um dos triângulos BDE e CDE estão na mesma razão de ADE.

Então o triangulo BDE é igual ao triangulo CDE (proposição 9 do livro V);.e eles estão sobre a mesma base $D E$.

Mas os triângulos iguais que estão sobre a mesma base estão também na mesma paralela (proposição 39 do livro I) ${ }^{11}$.

Portanto DE é paralela a BC.

Portanto ...

C.Q.D.

\footnotetext{
${ }^{8}$ Id. Ibid. fl. 42

${ }^{9}$ Equal magnitudes have to the same the same ratio, as also has same to equal magnitudes. (HEATH, 1956, p. 148).

${ }^{10}$ If a first magnitude be the same multiple of a second that a third is of a fourth, and a fifth also be the same multiple of the second that a sixth is of the fourth, the sum of the first and fifth will also be the same multiple of the second that the sum of the third and sixth is of the fourth (HEATH, 1956, p. 139).

${ }^{11}$ Id Ibid fl. 42
} 


\section{ALGUNS LIVROS-TEXTO DE MATEMÁTICA DA ATUALIDADE}

\subsection{Números Racionais e Irracionais - Ivan Niven}

O teorema de Thales está apresentado no livro Números Racionais e Irracionais, na seção sobre uma aplicação à geometria, dentro do capítulo intitulado Números Reais. Encontramos seu enunciado no teorema 3.1: "Se três paralelas são cortadas por duas transversais, com ponto de intersecção $\mathrm{A}, \mathrm{B}, \mathrm{C}, \mathrm{A}^{\prime}, \mathrm{B}^{\prime}, \mathrm{C}^{\prime}$, como na Fig. 12, então $\frac{A B}{B C}=\frac{A^{\prime} B^{\prime}}{B^{\prime} C^{\prime}}$, onde, por exemplo, $\mathrm{AB}$ representa o comprimento do segmento determinado por A e B" (NIVEN, 1985, p. 72).

Figura 6. Demonstração.

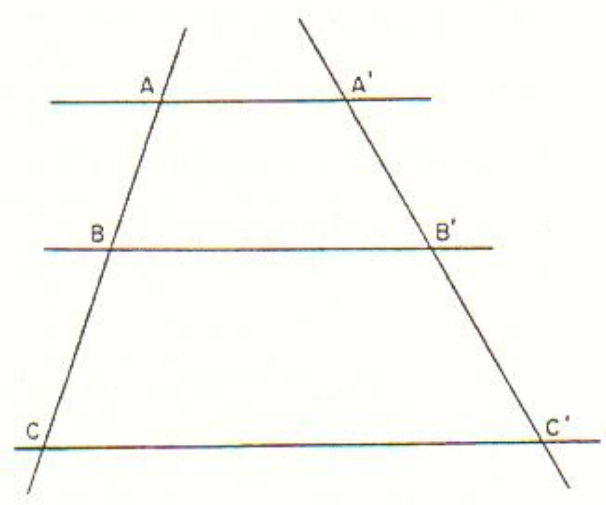

Figura 12

Fonte: Niven, 1985.

Em seguida, o autor menciona o uso do teorema na demonstração do teorema fundamental da semelhança, no teorema de Pitágoras e na Geometria Analítica. E explica que

Vamos, agora, provar o teorema 3.1 para o caso em que $A B / C D$ é irracional. Vamos aceitar a validade do Teorema 3.1 no caso de $A B / C D$ ser racional, pois esta parte do teorema é, em geral, demonstrada nos livros de Geometria Elementar. Antes de demonstrarmos o Teorema 3.1 para $\mathrm{AB} / \mathrm{BC}$ irracional, será útil estabelecer resultado preliminar:

Teorema 3.2. Se $\mathrm{m}$ e $\mathrm{n}$ forem inteiros positivos tais que $\frac{m}{n}<\frac{A B}{B C}$, então $\frac{m}{n}<\frac{A^{\prime} B^{\prime}}{B^{\prime} C^{\prime}}$. 
Esse resultado é demonstrado nesse livro, porém não iremos nos deter nessa demonstração. Como resultado, encontra-se, em seguida, o enunciado do corolário do Teorema 3.2: Se $\frac{m}{n}>\frac{A B}{B C}$, então $\frac{m}{n}>\frac{A^{\prime} B^{\prime}}{B^{\prime} C^{\prime}}$

Partindo desses resultados, o autor demonstra o teorema de Thales da seguinte maneira:

\section{DEMONSTRAÇÃO}

Seja $\beta$ um número irracional representando a razão $A B / B C$. Usaremos a representação decimal de $\beta$, como na Seç̧ão $3.2^{12}$.

Para ilustrar a passagem seguinte, façamos $\beta$ assumir o valor $\pi=3,14159 \ldots$, por exemplo. Podemos, então, escrever

(1)

$$
\begin{aligned}
& \frac{3}{1}<\beta<\frac{4}{1}, \\
& \frac{31}{10}<\beta<\frac{32}{10} \\
& \frac{314}{100}<\beta<\frac{315}{100} \\
& \frac{3141}{1000}<\beta<\frac{3142}{1000}, \ldots, \text { etc. }
\end{aligned}
$$

As frações, à esquerda, são obtidas, tomando os números 3; 3,1; 3,14; 3,141; da representação decimal de $\pi$. As frações do lado direito são obtidas, aumentando estes mesmos números de $1 ; 0,1 ; 0,01 ; 0,001$; etc.

A cadeia de desigualdade (1) é infinita; escrevemos somente as primeiras quatro. Estas desigualdades caracterizam o valor particular do $\beta$ em questão, isto é, caracterizam $\pi$. Ou seja, se um número $\beta$ satisfaz todas as desigualdades (1), então este número é igual a $\pi$.

As desigualdades (1) foram escritas em conexão com um exemplo ilustrativo, onde $\beta$ tinha o valor de $\pi$. Abandonaremos agora este exemplo, mas ressaltamos que, qualquer que seja o valor irracional que $\beta$ possa ter, sua representação decimal fornecerá uma cadeia de desigualdades

\footnotetext{
${ }^{12}$ Na seção 3.2 desse livro, o autor estuda a representação decimal para interpretar os números reais.
} 
(2)

$$
\begin{aligned}
\frac{a_{1}}{1}<\beta<\frac{1+a_{1}}{1}, \\
\frac{a_{2}}{10}<\beta<\frac{1+a_{2}}{10} \\
\frac{a_{3}}{100}<\beta<\frac{1+a_{3}}{100} \\
\frac{a_{4}}{1000}<\beta<\frac{1+a_{4}}{1000}, \ldots, \text { etc. }
\end{aligned}
$$

que caracterizará $\beta$ de modo único e, em cada desigualdade, $\beta$ estará entre dois números racionais. Os símbolos $\mathrm{a}_{1}, \mathrm{a}_{2}, \mathrm{a}_{3}, \ldots$ representam inteiros.

Nossa intenção é fazer $\beta^{\prime}$ representar a razão $A^{\prime} B^{\prime} / B^{\prime} C^{\prime}$ e demonstrar que $\beta^{\prime}$ também satisfaz as desigualdades (2), tal qual $\beta$. Mas, estas desigualdades caracterizam o número $\beta$ e, portanto, $\beta^{\prime}$ ficará identicamente $\operatorname{com} \beta$, de modo que

$$
\beta=\frac{\mathrm{AB}}{\mathrm{BC}}=\frac{A^{\prime} B^{\prime}}{B^{\prime} C^{\prime}}=\beta^{\prime}
$$

Só falta, então, demonstrar que $\beta^{\prime}$ satisfaz as desigualdades (2). Para isso usaremos o Teorema 3.2. Inicialmente, escolhamos qualquer um dos números $a_{1} / 1, a_{2} / 10, a_{3} / 100$, etc., digamos, $a_{3} / 100$ e interpretemos este como sendo o número racional $\mathrm{m} / \mathrm{n}$ do Teorema 3.2. Então, a hipótese do Teorema 3.2,

$$
\frac{m}{n}<\frac{A B}{B C} .
$$

se transforma em

$$
\frac{a_{3}}{100}<\beta,
$$

e isto é válido por causa das desigualdades (2). Logo, o Teorema 3.2 nos diz que

$$
\frac{m}{n}<\frac{A^{\prime} B^{\prime}}{B^{\prime} C^{\prime}}
$$

isto é,

$$
\frac{a_{3}}{100}<\beta^{\prime} .
$$

Vemos, assim, que $\beta^{\prime}$ satisfaz

$$
\frac{a_{1}}{1}<\beta^{\prime}, \frac{a_{2}}{10}<\beta^{\prime}, \frac{a_{3}}{100}<\beta^{\prime}, \frac{a_{4}}{1000}<\beta^{\prime}, \text { etc. }
$$

Fazendo um uso análogo do corolário do Teorema 3.2, obtemos as desigualdades $\beta^{\prime}<\frac{1+a_{1}}{1}, \beta^{\prime}<\frac{1+a_{2}}{10}, \beta^{\prime}<\frac{1+a_{3}}{100}, \beta^{\prime}<\frac{1+a_{4}}{1000}$, etc.

Portanto, $\beta^{\prime}$ satisfaz as desigualdades (2) tal qual $\beta$; logo $\beta=\beta^{\prime}$, o que completa a demonstração do teorema 3.1.

(NIVEN, 1985, p. 72-78) 


\subsection{Meu Professor de Matemática e outras histórias - Elon Lages Lima}

Para a prova do teorema de Thales, o autor, durante a demonstração, utilizará o teorema 1, porém não iremos demonstrá-lo, apenas enunciá-lo (LIMA, 2004, p. 129):

Teorema 1. As seguintes afirmações a respeito de $y=f(x)$ são equivalentes:

1) y é diretamente proporcional a $\mathrm{x}$;

2) para todo número real $c>0$, tem-se $f(c . x)=c . f(x)$;

3) existe um número k, chamado a "constante de proporcionalidade" entre $\mathrm{xe}$ $y$, tal que $f(x)=k \cdot x$ para todo $x$.

Segue o enunciado e a demonstração do teorema de Thales:

Teorema 3: Toda paralela a um dos lados de um triângulo divide os outros dois lados em segmentos proporcionais.

Figura 7. Demonstração.

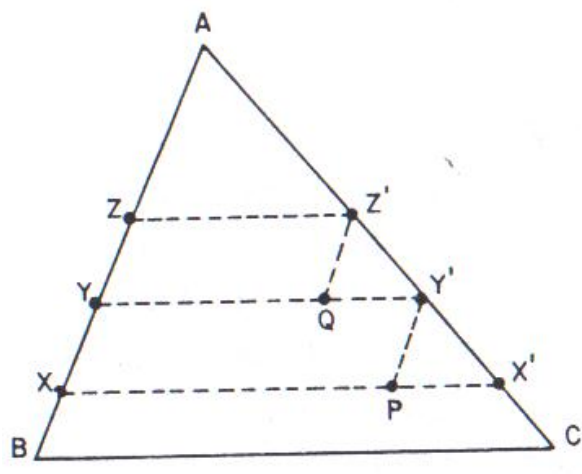

Figura 1.

Demonstração: Seja $A B C$ o triângulo. $A$ cada ponto $X$ do lado $A B$ façamos corresponder o ponto $\mathrm{X}^{\prime}$ do lado $\mathrm{AC}$, de tal modo que $\mathrm{XX}^{\prime}$ seja paralela a $\mathrm{BC}$. Provaremos que o comprimento $X^{\prime} C$ é diretamente proporcional ao comprimento $X B$. Em primeiro lugar, é claro que se $X, Y$ são pontos de $A B$ tais que $X B<Y B$ então $X^{\prime} B<Y^{\prime} B$ porque $X X^{\prime}$ e $Y Y^{\prime}$ são paralelos. Em seguida, afirmamos que se os pontos $X, Y, Z$ do lado $A B$ são tais que $X Y=Y Z$ então $X^{\prime} Y^{\prime}=Y^{\prime} Z^{\prime}$. Para ver isto, tomemos os pontos $P$ em $X X^{\prime}$ e $Q$ em $Y Y^{\prime}$ de modo que $Y^{\prime} P$ e $Z^{\prime} Q$ sejam paralelas a $A B$. Os triângulos $P X^{\prime} Y^{\prime} e{ }^{\prime} Y^{\prime} Z^{\prime}$ são congruentes porque têm um lado igual $\left(P Y^{\prime}=Q Z^{\prime}\right)$ compreendido entre ângulos iguais. Desta observação resulta que se $X, Y$ são 
pontos de $A B$ com $Y B=n \cdot X B$ então seus correspondentes $X^{\prime}, Y^{\prime}$ no lado $A C$ são tais que $Y^{\prime} C=n \cdot X^{\prime} C$. Isto conclui a verificação de que o comprimento $X^{\prime} C$ é diretamente proporcional a XB. Pelo Teorema 1, existe uma constante $\mathrm{k}$ tal que, para todo ponto $X$ do segmento $A B$, tem-se $X^{\prime} C=k . X B(1)$. Em particular, para $X=A$, como $A^{\prime}=A$, obtemos $A C=k \cdot A B(2)$. Subtraindo (1) de (2) vem: $A X^{\prime}=k \cdot A X(3)$. Dividindo a igualdade (3) pela igualdade (1) resulta

$$
\frac{A X^{`}}{X^{`} C}=\frac{A X}{X B}
$$

Isso é precisamente o que estipula o Teorema de Tales.

(LIMA, 2004, p. 132-133)

Em seguida o autor faz uma observação que "o teorema de Tales equivale a afirmar que $X ` C$ é diretamente proporcional a XB. O leitor interessado poderá verificar que a constante de proporcionalidade $k=A C / A B$ é igual a senB/senC" (LIMA, 2004, p. 133).

\subsection{Geometria Moderna: Parte I - Moise e Dowrs}

Nesse livro, Geometria Moderna, o autor nomeia como teorema fundamental sobre proporcionalidade o nosso conhecido teorema de Thales, demonstrando-o por meio do método das áreas, em um nível bem elementar. Segue a enunciação e a demonstração:

Teorema 12-1. O teorema Fundamental sobre Proporcionalidade.

Se uma reta paralela a um lado de um triângulo intercepta os outros dois lados em pontos distintos, então ela determina segmentos que são proporcionais a esses lados.

\section{DEMONSTRAÇÃO}

Figura 8. Demonstração.

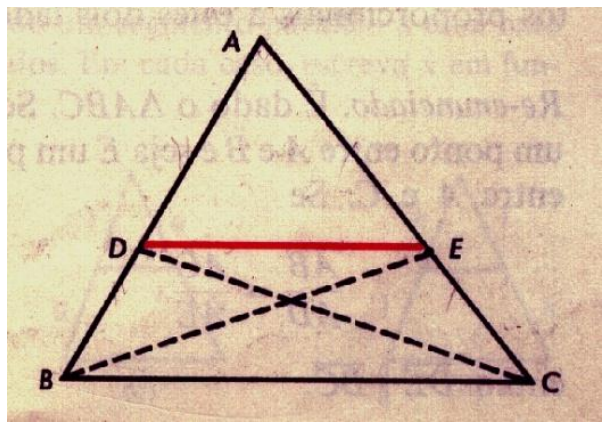


Re-enunciado. No $\triangle \mathrm{ABC}$ sejam $\mathrm{D}$ e $\mathrm{E}$ pontos de $\overline{\mathrm{AB}}$ e $\overline{\mathrm{AC}}$ tais que $\overline{\mathrm{DE}} / / \overline{\mathrm{BC}}$. Então $\frac{A B}{A D}=\frac{A C}{A E}$.

\section{Demonstração $^{13}$}

Nos $\triangle \mathrm{ADE}$ e $\triangle \mathrm{BDE}$ consideremos $\overline{\mathrm{AD}}$ e $\overline{\mathrm{BD}}$ como as bases. Então esses triângulos têm a mesma altura. (Por que?) Portanto, pelo Teorema $11-7^{14}$, a razão de suas áreas é igual a razão de suas bases e temos

(1) $\frac{a \triangle B D E}{a \triangle A D E}=\frac{B D}{A D}$.

Figura 9. Demonstração.

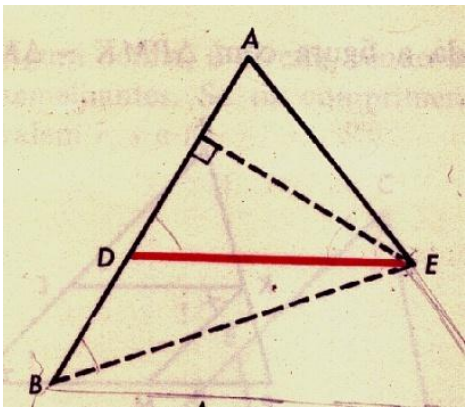

Analogamente, nos $\triangle \mathrm{ADE}$ e $\triangle \mathrm{CDE}$ consideramos $\overline{A E}$ e $\overline{C E}$ como bases. Como esses triângulos têm mesma altura, concluímos, como antes, que

(2) $\frac{\mathrm{a} \triangle \mathrm{CDE}}{\mathrm{a} \triangle \mathrm{ADE}}=\frac{\mathrm{CE}}{\mathrm{AE}}$

Figura 10. Demonstração.

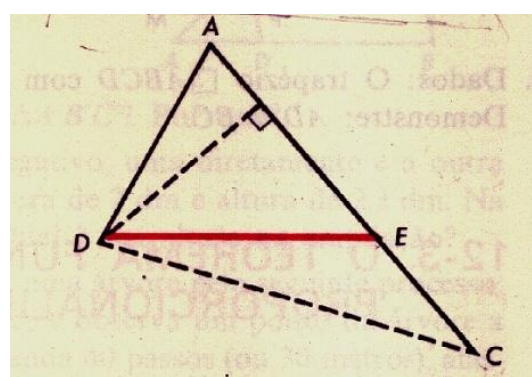

Mas $\triangle \mathrm{BDE}$ e $\triangle \mathrm{BDE}$ têm a mesma base $\overline{D E}$. (veja a figura à direita do reenunciado). E eles têm a mesma altura, porque $\overleftrightarrow{D E}$ e $\overleftrightarrow{B C}$ são paralelas. Portanto, pelo Teorema 11-6.

(3) $\triangle \mathrm{BDE}=\triangle \mathrm{CDE}$

\footnotetext{
${ }^{13} \mathrm{O}$ tratamento que o autor dá, por exemplo, a $\mathrm{a} \triangle \mathrm{BDC}$ é a área do triângulo $\mathrm{BDC}$.

${ }^{14}$ Se dois triângulos têm a mesma altura $h$, então a razão entre suas áreas é igual a razão entre suas bases (MOISE, 1971: 281)
} 
Combinado as três equações (1), (2) e (3), obtemos

(4) $\frac{B D}{A D}=\frac{C E}{A E}$.

Adicionando 1 a ambos os membros da equação (4), obtemos

(5) $\frac{B D+A D}{A D}=\frac{C E+A E}{A E}$, ou $\frac{A B}{A D}=\frac{A C}{A E}$,

como queríamos demonstrar.

O recíproco do Teorema Fundamental sobre Proporcionalidade é muito fácil de demonstrar.

(MOISE e DOWRS, 1971p. 307-308)

\section{CONSIDERAÇÕES FINAIS}

Nas demonstrações apresentadas acima, retirada de famosos livros didáticos voltados para o ensino superior, e outros utilizados como leitura complementar para o Ensino Médio, percebemse três maneiras distintas de provar o teorema de Thales. Na demonstração apresentada no livro de Niven (1985), é importante destacar a não utilização de conceito de comensurabilidade de segmentos. Ele conduz a demonstração por meio de definições de número racional e irracional, utilizando a representação decimal.

No texto de Lima (2004), o autor também não utiliza conceitos envolvendo comensurabilidade de segmentos. Ele emprega, para a demonstração, resultados como constante de proporcionalidade, grandezas proporcionais, entre outros.

Na última demonstração apresentada, Moise e Dowrs (1971) também não utilizam conceitos envolvendo comensurabilidade de segmentos, porém possibilitam ao leitor uma prova bastante semelhante à que podemos encontrar no livro VI dos "Os Elementos" de Euclides, utilizando a comparação entre áreas.

É pertinente observar que cada autor teve um objetivo ao propor essas demonstrações, em que o teorema não estava relacionado diretamente com o conteúdo da disciplina de Geometria. Niven (1985), por exemplo, demonstrar-o dentro do estudo com os números reais. 
Desse modo, no estudo do teorema de Thales, encontramos outras formas de demonstração que não trabalham diretamente com a comensurabilidade de segmentos, de modo que possibilite caminhos diferentes para a abordagem do teorema em vários níveis de ensino, cabendo ao professor escolher os conceitos compatíveis com seus objetivos.

\section{REFERÊNCIAS}

BICUDO, I. EUCLIDES, Os Elementos. São Paulo: Editora Unesp, 2009.

BOYER, C. História da Matemática. 2. ed. São Paulo: Edgard Blücher, 1998.

EVES, F. Introdução à História da Matemática. Campinas, SP: Editara da UNICAMP, 2004.

F.I.C. Elementos de Geometria. Rio de Janeiro: Livraria Garnier, 1923.

FOWLER, D. H. The Mathematics of Plato's Academy - a New Reconstruction. 2. ed. Oxford: Claredon Press, 1999.

HARUNA, N. C. A. Teorema de Thales: uma abordagem do processo ensino aprendizagem. 2000. 230f. Dissertação (Mestrado em Educação Matemática) - Pontifícia Universidade Católica, São Paulo, 2000.

HEATH, T. L. The Thirteen Books of Euclid's Elements. v.2, 2nd. Ed, New York:Dover Publications, 1956.

LIMA, E. L. Meu Professor de Matemática e outras histórias. 4ạ edição. Rio de Janeiro: SBM, 2004. (Coleção Professor de Matemática).

LINTZ, R. G. História da Matemática. Blumenal: Editora FURB, v. 1, 1999.

MASSOT, C. Autour de Thales. Commission Inter-IREM Premier Cycle, I'Imprimerie CRIRAT, 1995.

MOISE, E. E.; DOWRS, F. L. Geometria Moderna - Parte I. Renata G. Watanabe e Dorival A. Mello. São Paulo: Edgard Blücher, 1971.

NIVEN, I. Números: Racionais e Irracionais. Tradução de Renate Watanabe. Rio de Janeiro: SBM, 1984. (Coleção Fundamentos da Matemática Elementar).

QUINTELLA, A. Matemática para terceira série ginasial. Curso Ginasial. 59. ed. Rio de Janeiro: Companhia Editora Nacional, 1963. 